\title{
mRNA Transport in Dendrites: RNA Granules, Motors, and Tracks
}

\author{
Nobutaka Hirokawa \\ Department of Cell Biology and Anatomy, Graduate School of Medicine, University of Tokyo, Hongo, Tokyo 113-0033, Japan
}

The targeting of mRNAs to neuronal dendrites is an important protein sorting mechanism. Recent studies have revealed that mRNAs are transported by molecular motors. The kinesin superfamily protein KIF5 transports mRNAs such as calcium/calmodulin-dependent kinase II $\alpha$ (CaMKII $\alpha)$ and Arc mRNAs along microtubules in large granules containing proteins involved in RNA transport, protein synthesis, RNA helicases, heterogeneous nuclear ribonucleoproteins (hnRNPs), and RNA-associated proteins. This transport is fundamental to local protein synthesis and to the regulation of neuronal function.

Key words: microfilament; tubulin; transport; granule; RNA trafficking; intracellular signaling; cytoskeleton

\section{Intracellular transport in neurons}

Neurons have a highly polarized cell structure and typically contain one long, thin filamentous axon and multiple dendrites that differ from one another morphologically and functionally. Axons and dendrites differ in their intracellular signaling cascades and trafficking pathways. A consensus view is that proteins destined for axons and presynaptic terminals are synthesized in the cell body and transported down the axon in membranous organelles or protein complexes. Whereas most proteins destined for dendrites and dendritic spines are conveyed from the cell body, a subset of mRNAs are transported into dendrites to support local protein synthesis (Steward and Worley, 2001; Grossman et al., 2006; Martin and Zukin, 2006; Pfeiffer and Huber, 2006; Schuman et al., 2006; Wells, 2006). Selective sorting of mRNAs to axons and dendrites has been the focus of much recent attention. This review focuses on the motors that mediate selective transport of RNAs into dendrites and axons and the molecular components of RNA-transporting granules.

In axons and dendrites, microtubules run in a longitudinal direction and serve as tracks for the transport of membranous organelles and macromolecular complexes. Microtubules in axons and distal dendrites are unipolar, i.e., the plus end (the fast growing end) points in the direction of the synapse. In contrast, microtubules in proximal dendrites are of mixed polarity. Molecular motors of the kinesin and dynein superfamilies actively move along microtubules carrying various cargoes (Hirokawa, 1998; Hirokawa and Takemura, 2005). Both kinesin and dynein superfamily proteins are microtubule-dependent motors that slide along microtubules. The large kinesin superfamily includes

\footnotetext{
Received April 29, 2006; revised May 26, 2006; accepted May 26, 2006.

This work was supported by a Center of Excellence Grant from the Ministry of Education, Culture, Sports, Science, and Technology of Japan. I thank H. Fukuda for secretarial assistance.

Correspondence should be addressed to Dr. Nobutaka Hirokawa, Department of Cell Biology and Anatomy, Graduate School of Medicine, University of Tokyo, Hongo, Tokyo 113-0033, Japan. E-mail: hirokawa@m.u-tokyo.ac.jp.

DOI:10.1523/JNEUROSCI.1821-06.2006

Copyright $\odot 2006$ Society for Neuroscience $\quad 0270-6474 / 06 / 267139-04 \$ 15.00 / 0$
}

45 members in mice and humans. Whereas kinesin superfamily proteins (KIFs) typically move toward the plus end of microtubules and participate in anterograde transport, cytoplasmic dyneins are minus-end-directed motors and mediate retrograde transport from axonal or dendritic terminals to the cell body.

Many membranous organelles are transported from the cell body and down the axon to the presynaptic terminal. KIF1A and KIF1B $\beta$ transport synaptic vesicle precursors containing synaptic vesicle proteins such as synaptotagmin, synaptophysin, and Rab 3A. KIF1B $\alpha$ and KIF5 (KIF5A, KIF5B, and KIF5C) transport mitochondria in the anterograde direction. KIF5 also transports other cargoes, including vesicles that contain APPs (amyloid precursor proteins) and vesicles containing APOER2 (apolipoprotein $\mathrm{E}$ receptor 2) through interactions with JIPs (JNKinteracting proteins) and kinesin light chain (KLC). KIF5 also participates in slow axonal transport of cytoskeletal proteins. KIF3, mainly composed of KIF 3A/KIF3B heterodimers and an associated protein KAP3, transports vesicles associated with fodrin and is important for neurite extension. Recent studies involving a conditional knock-out of the KAP3 subunit of KIF3 impairs transport of $\mathrm{N}$-cadherin and $\beta$-catenin-containing vesicles from the Golgi to the cell surface of progenitor cells and causes abnormal proliferation of mouse neural progenitor cells (Teng et al., 2005; Hirokawa and Takemura, 2005).

In dendrites, transport of NMDA receptors is mediated by the molecular motor KIF17; binding is mediated via the scaffolding proteins mLin-7 (MALS), mLin-2 (CASK), and mLin-10 (Mint1), which form a macromolecular complex containing the NMDA receptor NR2B subunit (Guillaud et al., 2003) (Fig. 1). Overexpression of KIF17 enhances spatial and working memory in transgenic mice (Wong et al., 2002). Moreover, the genes for KIF17 and NR2B are coregulated, such that overexpression of KIF17 leads to the upregulation of NR2B. This process might depend on increased phosphorylation of the transcription factor CREB. AMPA glutamate receptors, in contrast, are transported by the conventional motor KIF5; in this case binding is mediated via the adaptor/scaffolding protein, GRIP1 (glutamate receptor- 
interacting protein 1) and kinesin heavy chains (Hirokawa and Takemura, 2005) (Fig. 1).

\section{mRNA transport in dendrites}

In many cell types, but especially neurons, the targeting of mRNAs to distinct cellular compartments is an important proteinsorting mechanism (Grossman et al., 2006; Martin and Zukin, 2006; Pfeiffer and Huber, 2006; Schuman et al., 2006; Wells, 2006). Although the majority of mRNAs are localized to the cell body, a subset of mRNAs is transported to dendrites. Dendritic protein synthesis can occur during plasticity and may produce long-lasting changes in synaptic strength (Kang and Schuman, 1996; Huber et al., 2000). Several proteins associated with RNA transport have been extensively studied, such as hnRNP-A2, staufens, and familial mental retardation proteins (FMRPs; FMR1, FXR1, and FXR2), as have the mRNAs being transported, such as $\beta$-actin (Bassell et al., 1998), MAP2 (microtubule-associated protein) (Blichenberg et al., 1999), CaMKII $\alpha$ (Blichenberg et al., 2001), and Arc (Lyford et al., 1995). Recently, staufenenriched RNA-containing granules have been isolated (Krichevsky and Kosik, 2001; Mallardo et al., 2003), and one by one the proteins involved in RNA transport have been identified, including Pur $\alpha$, Me31B, and ZBP1 (Nakamura et al., 2001; Zhang et al., 2001; Ohashi et al., 2002). However, comprehensive information about the RNA-transporting granules or the relationships among the associated proteins has not been published. It has been reported that RNA transport requires microtubuledependent motors (kinesin and dynein) (Brendza et al., 2000; Carson et al., 2001), but until recently the mechanism by which motor proteins transport RNAs has remained elusive.

\section{mRNA-associated granules and kinesin motors}

Recently, Hirokawa and colleagues isolated a large detergentresistant, RNase-sensitive granule $(\sim 1000 \mathrm{~S}$ in size $)$ from mouse brain as a binding partner of conventional kinesin KIF5 (Kanai et al., 2004). Altogether, 42 proteins were identified in the granules, of which 12 were extensively characterized: Pur $\alpha$, a protein associated with RNA transport, and its family protein, $\operatorname{Pur} \beta$; eight RNA-associated proteins (hnRNP-U, PSF, DDX1, DDX3, SYNCRIP, TLS, NonO, and ALY); and two proteins (HSPC117 and CGI-99), whose functions remain unknown. The granules also contained Staufen, FMRPs, EF- $1 \alpha$, and the mRNAs for CaMKII $\alpha$ and Arc (Kanai et al., 2004). The proteins were classified according to their binding affinities for KIF5, and direct interactions between components of the granules were determined by coimmunoprecipitation analysis. In cultured hippocampal neurons, the identified protein components of the granules colocalized with Pur $\alpha$ and KIF5 and these granules were transported bidirectionally in dendrites, indicating a tug-of-war between kinesin and opposing motors such as dynein. The distally directed movement of the granules was enhanced by the overexpression of KIF5 and reduced by inhibition of KIF5, thereby confirming the role of kinesin in transport of RNA granules. A two-dimensional (2D)-PAGE study followed by mass spectrometry/mass spectrometry (MS/MS) analysis (2D-MS/ MS) revealed a total of 42 proteins, including proteins involved in RNA transport, protein synthesis, hnRNPs, RNA helicases, and translational silencing (Kanai et al., 2004).

RNA-containing granules that are transported to dendrites bind to the C-terminal tail of KIF5. The minimal binding site for RNA-containing granules is a 59 aa sequence (residues 865-923), which is conserved in KIF5A, KIF5B, and KIF5C. Among the proteins in large RNA-containing granules, $\operatorname{Pur} \alpha$ and $\operatorname{Pur} \beta$ were the two most strongly bound, as determined by sequential elution in the pull-down assay by stepwise increases in salt concentration. However, the direct binding partner of KIF5 remains unknown. When cultured neurons were transfected with both green fluorescent protein (GFP)-Pur $\alpha$ and cyan fluorescent protein (CFP)-KIF5, Pur $\alpha$-containing granules were transported exclusively to dendrites, although CFP-KIF5 is distributed to both axons and dendrites. However, a CFP-tagged dominant-negative KIF5 mutant $(\mathrm{CFP}-\delta \mathrm{N} 1)$, which contains the C-terminal tail RNA granule-binding site but lacks the N-terminal motor domain inhibited the movement of RNA-containing granules toward distal dendrites. CaMKII $\alpha$ mRNA and Arc mRNA colocalized with Pur $\alpha$-containing granules, but tubulin mRNA did not. RNA interference of the component proteins of RNA-containing granules such as hnRNPU suppressed transport of Staufen, Pur $\alpha$, and polypyrimidine tract-binding protein-associated splicing factor (PSF). These results show that transport of RNAcontaining granules to dendrites requires direct binding to the C-terminal tail of KIF5 (Kanai et al., 2004).

Using HEK (human embryonic kidney) 293 cells, components of Staufen1-containing particles were affinity purified (Brendel et al., 2004). The results imply that in the cytoplasm of mammalian cells, an association with the ribosomal P stalk protein PO recruits Staufen1 into a ribosome-containing ribonucleoprotein particle that also contains kinesin, protein phosphatase 1, and nucleolin (Brendel et al., 2004). Similarly, hStaufencontaining complexes were purified by affinity chromatography 
from human cells transfected with a TAP-tagged hStaufen gene. By mass spectrometry, the complex contained ribosomes and proteins involved in the control of protein synthesis [PABP1 (poly(A)-binding protein) and FMRP], cytoskeletal proteins (tubulins, tau, actin, and internexin), cytoskeleton control proteins (IQGAP1, cdc42, and rac1), motor proteins (dynein, kinesin, and myosin), and proteins normally found in the nucleus such as nucleolin and RNA helicase A (Villacé et al., 2004). Bannai et al. (2004) reported that SYNCRIP (heterogenous nuclear ribonuclear protein Q1/NSAP1) was transported bidirectionally within dendrites, at a velocity about equivalent to that of Pur $\alpha$ containing granules (Kanai et al., 2004). SYNCRIP colocalized in cultured neurons with Staufen 1 and the $3^{\prime}$ - untranslated region of inositol 1,4,5-triphosphate receptor type $1 \mathrm{mRNA}$, indicating that it is transported as a component of mRNA granules (Bannai et al., 2004)

In Drosophila melanogaster oocytes, oskar mRNA is localized to the posterior pole. Localization of oskar depends on the kinesin heavy chain (KIF5) and not the KLC, as shown in a KLC null mutant (Brendza et al., 2000). In addition, in an established $D$. melanogaster Schneider 2 (S2) cell line, GFP-tagged FMRP forms ribonucleoprotein granules that move along the long thin processes. The movement of the granules depends on KIF5, but not KLC, as shown by RNA-mediated interference of KLC. These results indicate that transport of mRNAs in these cells depends on KIF5 and not KLC (Ling et al., 2004). Recently it was shown that an RNA-binding protein, TLS (translocated in liposarcoma) is transported to dendrites, and that it is recruited to dendrites not only via microtubules, but also via actin filaments (Fujii et al., 2005). This finding suggests that not only KIF5, but also myosinbased motors, participate in the proper targeting of mRNA and associated proteins in dendrites.

\section{Conclusion and future perspectives}

In summary, it has become clear that certain mRNAs such as CAMKII $\alpha$ mRNA and Arc mRNA are transported in dendrites in granules (>1000 S), large macromolecular complexes. These granules are transported by KIF5, which binds RNA-binding proteins by a recognition motif in its tail domain (Fig. 1). Other motors such as dynein and myosin may also be involved in transport of RNA-containing granules. Although the polarity of microtubules in proximal dendrites is mixed, the polarity of microtubules in distal dendrites is the same as in the axon. Thus, anterograde and retrograde transports could be performed by KIF5 and dynein, respectively, in dendrites. Because actin filaments are the main cytoskeletal elements in the spine shaft, myosin may function to transport mRNAs and granules locally within the spine. Thus, the transport of mRNA-containing protein complexes by motor proteins is fundamental to local protein synthesis and to the regulation of neuronal functions

With regard to the mechanism of mRNA transport in dendrites, an immediate and important question is "What is the direct binding partner for KIF5 in the granules?" The answer to this question will provide a basis for understanding the regulation of mRNA transport, and also for activity-dependent regulation of local protein synthesis. Another interesting question is how directional transport of mRNA is regulated, because kinesin can transport cargoes both to the axon and dendrites, whereas mRNA and associated granules are mainly conveyed to dendrites. In this respect, it is noteworthy that the KIF5 motor domain by itself recognizes the characteristics of microtubules in the initial segment of the axon and tends to preferentially direct toward the axon (Nakata and Hirokawa, 2003), whereas overexpression of
KIF5 enhances transport of Pur $\alpha$ granules in dendrites, not in the axon (Kanai et al., 2004). These observations suggest the possibility that binding of cargoes such as mRNA-protein complexes to the tail domain of KIF5 steers kinesin motors toward dendrites. Dendritic targeting cis-acting elements, although variant, have been identified in many neuronal mRNAs (Blichenberg et al., 1999, 2001). These cis-acting elements could control the binding of mRNAs to granules and the mechanism of selective transport to dendrites may be mainly determined via interactions among microtubules, KIFs, and granules. Finally, understanding the functions of the various protein components of RNA granules will be fundamental to elucidating the functional significance of mRNA transport in dendrites.

\section{References}

Bannai H, Fukatsu K, Mizutani A, Natsume T, Iemura S, Ikegami T, Inoue T, Mikoshiba K (2004) An RNA-interacting protein, SYNCRIP (Heterogeneous Nuclear Ribonuclear Protein Q1/NSAP1) is a component of mRNA granule transported with inositol 1,4,5-trisphosphate receptor type 1 mRNA in neuronal dendrites. J Biol Chem 279:53427-53434.

Bassell GJ, Zhang H, Byrd AL, Femino AM, Singer RH, Taneja KL, Lifshitz LM, Herman IM, Kosik KS (1998) Sorting of beta-actin mRNA and protein to neurites and growth cones in culture. J Neurosci 18:251-265.

Blichenberg A, Schwanke B, Rehbein M, Garner CC, Richter D, Kindler S (1999) Identification of a cis-acting dendritic targeting element in MAP2 mRNAs. J Neurosci 19:8818-8829.

Blichenberg A, Rehbein M, Muller R, Garner CC, Richter D, Kindler S (2001) Identification of a cis-acting dendritic targeting element in the mRNA encoding the alpha subunit of $\mathrm{Ca} 2+/$ calmodulin-dependent protein kinase II. Eur J Neurosci 13:1881-1888.

Brendel C, Rehbein M, Kreienkamp HJ, Buck F, Richter D, Kindler S (2004) Characterization of Staufen 1 ribonucleoprotein complexes. Biochem J 384:239-246.

Brendza RP, Serbus LR, Duffy JB, Saxton WM (2000) A function for kinesin $\mathrm{I}$ in the posterior transport of oskar mRNA and Staufen protein. Science 289:2120-2122.

Carson JH, Cui H, Barbarese E (2001) The balance of power in RNA trafficking. Curr Opin Neurobiol 11:558-563.

Fujii R, Okabe S, Urushido T, Inoue K, Yoshimura A, Tachibana T, Nishikawa T, Hicks GG, Takumi T (2005) The RNA binding protein TLS is translocated to dendritic spines by mGluR5 activation and regulates spine morphology. Curr Biol 15:587-593.

Grossman AW, Aldridge GM, Weiler IJ, Greenough WT (2006) Local protein synthesis and spine morphogenesis. J Neurosci 26:7151-7155.

Guillaud L, Setou M, Hirokawa N (2003) KIF17 dynamics and regulation of NR2B trafficking in hippocampal neurons. J Neurosci 23:131-140.

Hirokawa N (1998) Kinesin and dynein superfamily proteins and the mechanism of organelle transport. Science 279:519-526.

Hirokawa N, Takemura R (2005) Molecular motors and mechanisms of directional transport in neurons. Nat Rev Neurosci 6:201-214.

Huber KM, Kayser MS, Bear MF (2000) Role for rapid dendritic protein synthesis in hippocampal mGluR-dependent long-term depression. Science 288:1254-1257.

Kanai Y, Dohmae N, Hirokawa N (2004) Kinesin transports RNA: isolation and characterization of an RNA-transporting granule. Neuron 43:513-525.

Kang H, Schuman EM (1996) A requirement for local protein synthesis in neurotrophin-induced hippocampal synaptic plasticity. Science 273:1402-1406.

Krichevsky AM, Kosik KS (2001) Neuronal RNA granules: a link between RNA localization and stimulation-dependent translation. Neuron 32:683-696.

Ling SC, Fahrner PS, Greenough WT, Gelfand VI (2004) Transport of Drosophila fragile $\mathrm{X}$ mental retardation protein-containing ribonucleoprotein granules by kinesin-1 and cytoplasmic dynein. Proc Natl Acad Sci USA 101:17428-17433.

Lyford GL, Yamagata K, Kaufmann WE, Barnes CA, Sanders LK, Copeland NG, Gilbert DJ, Jenkins NA, Lanahan AA, Worley PF (1995) Arc, a growth factor and activity-regulated gene, encodes a novel cytoskeletonassociated protein that is enriched in neuronal dendrites. Neuron 14:433-445. 
Mallardo M, Deitinghoff A, Muller J, Goetze B, Macchi P, Peters C, Kiebler MA (2003) Isolation and characterization of Staufen-containing ribonucleoprotein particles from rat brain. Proc Natl Acad Sci USA 100:2100-2105.

Martin KC, Zukin RS (2006) RNA trafficking and local protein synthesis in dendrites: an overview. J Neurosci 26:7131-7134.

Nakamura A, Amikura R, Hanyu K, Kobayashi S (2001) Me31B silences translation of oocyte-localizing RNAs through the formation of cytoplasmic RNP complex during Drosophila oogenesis. Development 128:3233-3242.

Nakata T, Hirokawa N (2003) Microtubules provide directional cues for polarized axonal transport through interaction with kinesin motor head. J Cell Biol 162:1045-1055.

Ohashi S, Koike K, Omori A, Ichinose S, Ohara S, Kobayashi S, Sato TA, Anzai $\mathrm{K}$ (2002) Identification of mRNA/protein (mRNP) complexes containing Pur alpha, mStaufen, fragile X protein, and myosin Va and their association with rough endoplasmic reticulum equipped with a kinesin motor. J Biol Chem 277:37804-37810.

Pfeiffer EM, Huber KM (2006) Current advances in local protein synthesis and synaptic plasticity. J Neurosci 26:7147-7150.
Schuman EM, Dynes J, Steward O (2006) Synaptic regulation of translation of dendritic mRNAs. J Neurosci 26:7143-7146.

Steward O, Worley P (2001) Localization of mRNAs at synaptic sites on dendrites. Cell Differ 34:1-26.

Teng J, Rai T, Tanaka Y, Takei T, Nakata T, Hirasawa M, Kulkarni AB, Hirokawa N (2005) The KIF3 motor transports N-cadherin and organizes the developing neuroepithelium Nat Cell Biol 7:474-482.

Villacé P, Marión RM, Ortín J (2004) The composition of Staufencontaining RNA granules from human cells indicates their role in the regulated transport and translation of messenger RNA. Nucleic Acids Res 32:2411-2420.

Wells DG (2006) RNA-binding proteins: a lesson in repression. J Neurosci 26:7135-7138.

Wong RW-C, Setou M, Teng J, Takei Y, Horikawa N (2002) Overexpression of motor protein KIF17 enhances spatial and working memory in transgenic mice. Proc Natl Acad Sci USA 99:14500-14505.

Zhang HL, Eom T, Oleynikov Y, Shenoy SM, Liebelt DA, Dictenberg JB, Singer RH, Bassell GJ (2001) Neurotrophin-induced transport of a betaactin mRNP complex increases beta-actin levels and stimulates growth cone motility. Neuron 31:261-275. 